\title{
Influence and Its suppression of Swinging Antennas on Co-site Interference Cancellation System
}

\author{
Yunhao Jiang ${ }^{a}$, Wenfang Ding ${ }^{b}$, Ziqiang $\mathrm{Xi}^{\mathrm{c}}$, Nan Zhao ${ }^{\mathrm{d}}$ \\ ${ }^{1}$ Hubei Collaborative Innovation Center for High-efficiency Utilization of Solar Energy, \\ Hubei University of Technology Wuhan, Hubei Province, China \\ ${ }^{2}$ Hubei Key Laboratory for High-efficiency Utilization of Solar Energy and Operation Control \\ of Energy Storage System, Hubei University of Technology \\ Wuhan, Hubei Province, China \\ ajyh6858@126.com, b523661844@qq.com, ‘695936405@qq.com, d337652024@qq.com
}

\begin{abstract}
Keywords: co-site interference cancellation system (CICS); swing; modulation; interference cancellation ratio (ICR)

Abstract. This paper investigates the influence of swinging antennas on co-site interference cancellation system (CICS) and the improved interference cancellation method for keeping the effective interference cancellation as the antennas swing. The interference signal model coupled from transmitter to receiver was established when the antennas swing and the influence of swing antennas on received interference signal was analyzed. The swing of the antennas will cause the received interference signal to be modulated. The amplitude and the bandwidth of the interference signal will be changed. For suppressing the influence of swinging antennas on interference cancellation, the improved bandwidth designed method is proposed. Finally, the validity of the proposed method was confirmed by the simulation experiment.
\end{abstract}

\section{Introduction}

Nowadays, more and more electronic equipments were installed in common plat (such as war chariot, aircraft and warship). The mutual interferences of the antennas are serious due to the limited space isolation. For suppressing the interference in the Marine Corps' Expeditionary Fighting Vehicle (EFV), the Co-site Interference Cancellation System (CICS) was proved to be the best efficiency by testing many potential solutions [1]. The theoretical basis of the adaptive interference cancellation is the principle of adaptive noise cancellation and the LMS (least mean squares) algorithm mentioned by B. Widrow [2]. Reference [3] analyzed the performance of co-site interference cancellation system implemented by analogue circuit. The performance of CICS with integral control and low-pass control was analyzed in [4]. The improved method to decrease the impact of CICS on desired signal was proposed in [5]. Currently, the researches about the CICS are mainly under the condition that the antennas are stationary. But, in actual situations, some antennas (such as whip antennas) may swing with the moving of the carrier platform and the weather conditions. Here, the received interference signal will not be the same as the transmitting signal. Then, the interference cancellation performance of the CICS may drop because the correlation of the receiver interference and the reference signal is weakened. So, it is very important to research the influence of the swinging antennas on CICS and to propose a new method to guarantee the effective interference cancellation.

In this paper, the model of CICS is established firstly. Secondly, the received interference signal is modeled considering the swinging antennas and the influence of the swinging antennas on CICS is analyzed. Then, the method to suppress that influence is researched and proposed. Finally, the simulation is used to confirm the validity of the proposed method. 


\section{System Model.}

A typical CICS is shown in Fig. 1. The $X_{\mathrm{I}}(t)$ is the interference signal. The $X_{\mathrm{s}}(t)$ coupled from transmitting signal is reference signal. The $X_{\mathrm{s} 1}(t)$ and $X_{\mathrm{s} 2}(t)$ are two orthogonal reference signals with same amplitudes. The attenuator adjust the amplitude of the reference signal and output $Y_{1}(t)$ and $Y_{2}(t)$. $Y_{1}(t)$ and $Y_{2}(t)$ are then synthesized as the output signal $Y(t)$ via the synthesizer. $Y(t)$ is synthesized with the interference signal in the coupler and the receiving antenna for generating the cancellation residual signal $X_{\varepsilon}(t) . X_{\varepsilon}(t)$ is sent to the receiver and the correlator, respectively. The correlator completes the correlation operation of the reference signal and the cancelation residual signal. The weights $W_{1}(t)$ and $W_{2}(t)$ are the adjustment coefficients of the attenuators. The correlator consists of a multiplier and a low-pass filter. The low-pass filter can be represented as $k /(\tau \mathrm{s}+1)$, where $k$ denotes the gain of this filter and $\tau$ denotes the time constant. The block diagram of the CICS is shown in Fig. 2. For the moving of the carrier platform or the weather conditions, the antennas may swing. The coupled interference $X_{\mathrm{I}}(t)$ will vary with the swing of the antennas, but the reference signals will not, because they are coupled from the transmitter.

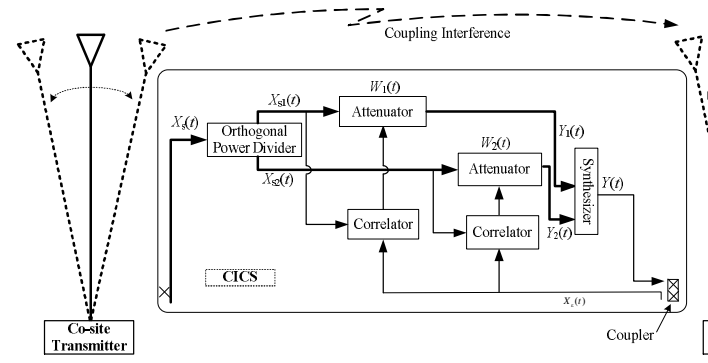

Figure 1. Cosite interference cancellation system.

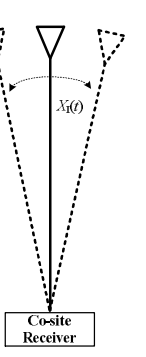

Figure 2. Block diagram of the cosite interference cancellation system.

Let orthogonal reference signals be

$$
X_{\mathrm{s} 1}(t)=E_{\mathrm{s}} \cos \left(\omega_{0} t\right), \quad X_{\mathrm{s} 2}(t)=E_{\mathrm{s}} \sin \left(\omega_{0} t\right)
$$

, the interference signal be

$$
X_{\mathrm{I}}(t)=E_{\mathrm{I}}(t) \cos \left(\omega_{0} t-\phi(t)\right)
$$

, where $E_{\mathrm{s}}$ and $E_{\mathrm{I}}(t)$ are the amplitudes of the reference signal and the received interference signal, respectively; $\omega_{0}$ is the angular frequency of the interference signal, $\Phi(t)$ is the phase angle of the received interference signal.

The residual error is

$$
X_{\varepsilon}(t)=X_{\mathrm{I}}(t)-\left[W_{1}(t) X_{\mathrm{s} 1}(t)+W_{2}(t) X_{\mathrm{s} 2}(t)\right]
$$

\section{Model of Interference Signal.}

From Fig. 1 and Fig. 2, if the antennas are stationary, the received interference signal is

$$
X_{\mathrm{I}}(t)=E_{\mathrm{I}} \cos (\omega t-\phi)
$$

, where the amplitude and the phase angle are constants. When the antennas swing, the coupling channel will change and the amplitude and the phase angle will vary over time randomly. Then the received interference signal becomes an amplitude modulation and phase modulation signal, as is shown in (2). But it is quite difficult to analyze the performance of the CICS thinking of the received interference as a random modulation signal. As the influence of swinging antennas on CICS is mainly the change of the received interference signal bandwidth and the order of the swing frequency is about several Hz, which is far less than that of the transmitting signal. Then, the received interference signal can be simplified. Thinking about the worst case, the antennas swing as a harmonic oscillation along radial and can swing to be horizontal. The oscillation frequency is about $10 \mathrm{~Hz}$.

Supposing the height of the antennas is $h$ and the distance of the antennas is $l$, then the minimum distance of the antennas is $l-2 h$ and the maximum distance is $l+2 h$. The received interference signal can be expressed as

$$
X_{\mathrm{I}}(t)=\left[1+m_{a} \cos \left(\omega_{\mathrm{s}} t\right)\right] E_{\mathrm{I}} \cos \left(\omega_{0} t-\left(1+m_{b} \cos \left(\omega_{\mathrm{s}} t\right)\right) \phi\right)
$$


, where $\mathrm{m}_{a}$ and $m_{b}$ are the modulation ratio of the amplitude and the phase angle, respectively; $\omega_{\mathrm{s}}=2 \pi f_{\mathrm{s}}$ is the swing angular frequency.

The isolation of the antennas can be written as [6]

$$
I S=22+20 \lg (L / \lambda)-\left(G_{\mathrm{t}}+G_{\mathrm{r}}\right)
$$

, where $L$ is the distance of the antennas, $\lambda$ is the wavelength of the transmitting signal, and $G_{\mathrm{t}}$ and $G_{\mathrm{r}}$ are gains of the transmitter antenna and receiver antenna, respectively. So, the maximum isolation and the minimum isolation can be expressed as

$$
I S_{\max }=22+20 \lg ((l+2 h) / \lambda)-\left(G_{t}+G_{r}\right), \quad I S_{\min }=22+20 \lg ((l-2 h) / \lambda)-\left(G_{t}+G_{r}\right) .
$$

From (6) and (7), as the antennas swing to be horizontal, the variations of isolation are

$$
\left\{\Delta I S_{\max }=I S_{\max }-I S=20 \lg (1+2 \mathrm{~h} / \mathrm{l}), \Delta I S_{\min }=I S_{\min }-I S=20 \lg (1-2 \mathrm{~h} / \mathrm{l})\right. \text {. }
$$

From (8), the modulation ratio $m_{a}$ can be written as

$$
m_{a \min }=\frac{2 h}{l+2 h} \stackrel{l>>2 h}{\rightarrow} m_{a \min } \approx \frac{2 h}{l}, m_{a \max }=\frac{2 h}{l-2 h} \stackrel{l>>2 h}{\rightarrow} m_{a \max } \approx \frac{2 h}{l} \text {. }
$$

When $l>>2 h$, the modulation ratio of amplitude can be approximated as $2 h / l$.

Thinking about the maximum swing, the maximum changed phase angle can be expressed as

$$
\left(1+m_{b}\right) \phi=\omega_{0} \frac{l+2 h}{c} \rightarrow m_{b}=2 h / l,\left(1-m_{b}\right) \phi=\omega_{0} \frac{l-2 h}{c} \rightarrow m_{b}=2 h / l
$$

, Where $c$ is the light speed and is about $3 \times 10^{8} \mathrm{~m} / \mathrm{s}$. So, from (9) and (10), the modulation ratios of amplitude and phase angle are the same. According to the characteristics of the phase modulated signal and (5), the maximum frequency shift is $\Delta \omega_{\mathrm{s}}=m_{b} \times \Phi \times \omega_{\mathrm{s}}$. Then, the bandwidth of the phase modulated signal is $B W_{\mathrm{p}}=2 m_{b} \times \Phi \times f_{\mathrm{s}}$. From (5), the bandwidth of the amplitude and phase modulated interference signal is

$$
B W_{\mathrm{s}}=2\left(m_{b} \phi+1\right) f_{\mathrm{s}}=2\left(m_{b} \cdot 2 \pi f_{0} l / c+1\right) f_{\mathrm{s}} .
$$

\section{Method to Suppress the Influence of Swing Antennas on CICS.}

As is shown in Fig. 2, the time-domain characteristics of CICS have been researched in [4, 5]. The transfer function of residual error to received interference can be written as

$$
H_{\varepsilon}(s)=\frac{X_{\varepsilon}(s)}{X_{\mathrm{I}}(s)}=\frac{G(s)}{1-G(s)}=\frac{\left[\tau\left(s-\mathrm{j} \omega_{0}\right)+1\right]\left[\tau\left(s+\mathrm{j} \omega_{0}\right)+1\right]}{\tau^{2} s^{2}+\left(2+k E_{\mathrm{s}}^{2}\right) \tau s+\left(k E_{\mathrm{s}}^{2}+1+\tau^{2} \omega_{0}^{2}\right)} .
$$

From(12), the frequency-domain characteristic of the CICS is shown in Fig. 3.

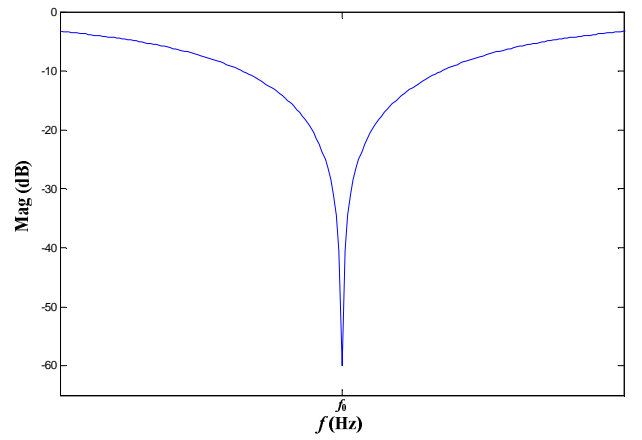

Figure 3. Amplitude frequency characteristic of CICS.

From Fig. 2 and Fig. 3, the CICS is a notch filter and the center frequency is the frequency of the reference signal. Though the reference signal is a single frequency signal, but the CICS has some notch bandwidth. It can cancel the signal with a certain bandwidth. As is shown in (11), though the received interference signal is modulated by the swinging antennas and its bandwidth is expanded, the expanded bandwidth is not big. So, the modulated received interference signal for the swinging antennas can be canceled effectively by proper design of the notch bandwidth.

Due to the requirement for protecting the receiver not to be block, supposing the ICR for unmodulated received interference signal as (4) is $I C R_{\mathrm{I}}$. For the side frequency signal amplitude of the 
phase modulated signal, which is needed to take into account, is about $10 \%$ of the amplitude of the unmodulated signal. So, the amplitude of the maximum side frequency signal is about $0.1 \times E_{\mathrm{I}}$ from (5). Then, the needed ICR of side frequency signal can be written as

$$
I C R_{\mathrm{Im}}=20 \lg \frac{0.1 E_{\mathrm{I}}}{E_{\mathrm{I}} /\left(10^{I C R_{\mathrm{I}} / 20}\right)}=20 \lg 0.1+I C R_{\mathrm{I}} .
$$

According to (12) and (13), the notch bandwidth corresponding to $I C R_{\operatorname{Im}}$ can be derived as $B W_{D} \approx 10^{-I C R_{\mathrm{Im}} / 20} \cdot k E_{\mathrm{s}}^{2} / 2 \pi \tau$.

In order to cancel the received interference signal effectively, (11) should be equal to (14), then

$$
k E_{\mathrm{s}}^{2} / 2 \pi \tau=B W_{\mathrm{s}} / 10^{-I C R_{\mathrm{Im}} / 20} \text {. }
$$

Equation (15) shows the received interference signal modulated by swinging antennas can be cancelled well by proper design of the gain $k$ and the time constant $\tau$. Provided the gain $k$ and the time constant $\tau$ are satisfied with (15), the CICS can ensure the effective interference cancellation as the antennas swing.

\section{The Simulation Analysis.}

Supposing $E_{\mathrm{s}}=1 \mathrm{~V}, E_{\mathrm{l}}=0.1 \mathrm{~V}, \tau=1 \mathrm{~s}, k=2000, \omega_{0}=2 \pi \times 2 \times 10^{6} \mathrm{rad} / \mathrm{s}, h=3 \mathrm{~m}, l=40 \mathrm{~m}, f_{\mathrm{s}}=10 \mathrm{~Hz}$. From (9) and (10), the amplitude and phase modulation ratios are $m_{a}=m_{b}=0.15$. Then the bandwidth of receiver interference is $B W_{\mathrm{s}} \approx 25 \mathrm{~Hz}$, according to (11).

If the designed ICR is about $60 \mathrm{~dB}$ when the antennas don't swing, then the residual error must be no more than $E_{\varepsilon}=E_{\mathrm{I}} / 10^{I C R_{1} / 20}=0.0001 \mathrm{~V}$.

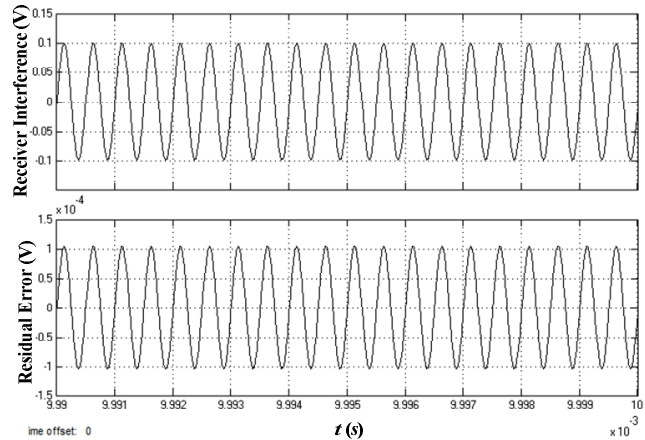

Figure 4. The receiver interference and residual error of CICS with the stationary antennas.

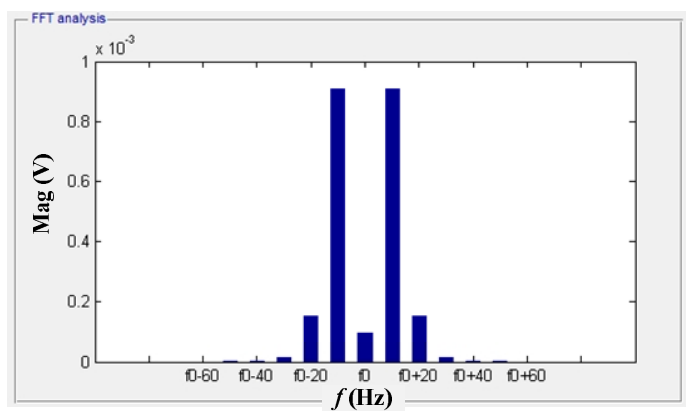

Figure 6. FFT analysis result of the residual error.

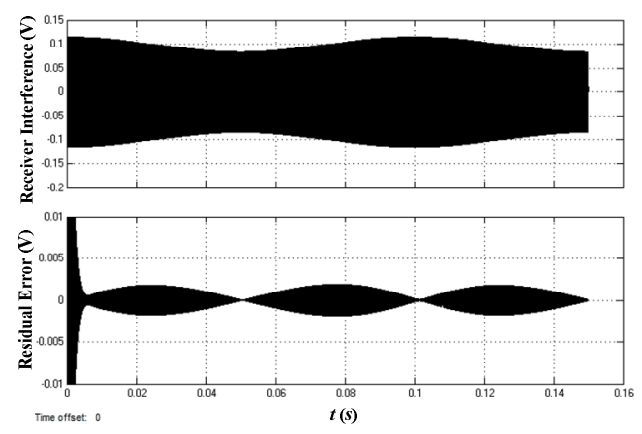

Figure 5. The receiver interference and residual error of CICS with the swinging antennas.

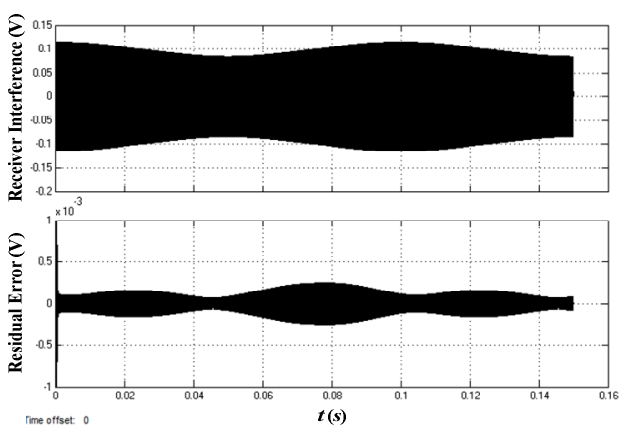

Figure 7. The receiver interference and residual error of the CICS with $\tau=0.1 \mathrm{~s}$.

Fig. 3 is the simulation results of CICS with the stationary antennas. From Fig. 3, the amplitude of the receiver interference is $0.1 \mathrm{~V}$ and the residual error is about $0.0001 \mathrm{~V}$. The ICR is $60 \mathrm{~dB}$ or so. It meets the design requirement.

If the antennas swing, the simulation results of CICS are shown in Fig. 4 and Fig. 5. As is shown in Fig. 5, the residual interference of the center frequency is about $0.0001 \mathrm{~V}$, which is the same as that of Fig. 3. The ICR of the center frequency is $60 \mathrm{~dB}$ and it meets the design requirement. But not all the residual interferences of side frequencies are less than $0.0001 \mathrm{~V}$. So, the time constant $\tau$ should be 
modified. According to (11), (13) and (15), the time constant $\tau$ can be calculated as about $0.1 \mathrm{~s}$. Then the simulation results of the CICS with $\tau=0.1 \mathrm{~s}$ are shown in Fig. 6 and Fig.7.

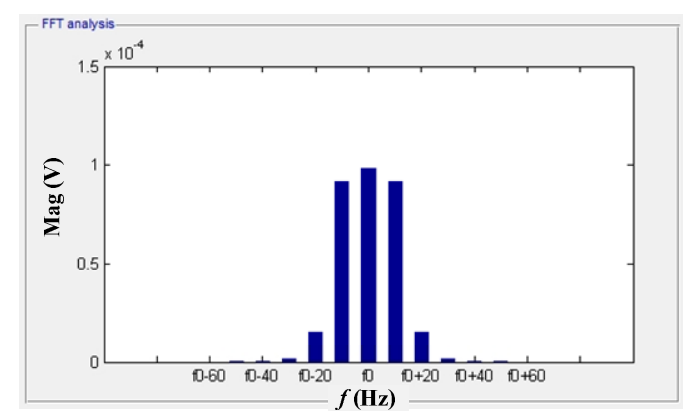

Figure 8. FFT analysis result of the residual error.

From Fig. 6 and Fig. 7, the frequency components of the residual interference are all less than $0.0001 \mathrm{~V}$. Their ICRs are all no less than $60 \mathrm{~dB}$. Therefore, the parameters design of the CICS meets the requirements for suppressing the influence of the swing antennas on interference cancellation effect.

\section{Conclusions.}

We have discussed the influence of swinging antennas on CICS. For the swing of the antennas, the receiver interference becomes an amplitude and phase modulation signal and the bandwidth of the receiver interference is expanded. Then, the effect of interference cancellation of CICS will decrease. By proposed improved design of time constant, the adverse effect can be overcome.

\section{Acknowledgements}

This work was financially supported by the Natural Science Foundation of Hubei Province (2016CFB396) and the High-Level Talent Foundation of Hubei University of Technology (BSQD2015021).

\section{References}

[1] Allsebrook, K. and Ribble, C. VHF cosite interference challenges and solutions for the United States Marine Corps' expeditionary fighting vehicle program. In Proceedings of the IEEE Military Communications Conference (Monterey, CA, USA, October 31 - November 03, 2004). 548-554.

[2] Widrow, B. Adaptive noise canceling: Principles and applications. Proc. IEEE. 63 (Dec. 1975), 1692-1716.

[3] Ma Y. G. and Du W. L. Performance analysis and design of adaptive interference canceling system. Journal of Xi'dian University. 1 (Jan. 1996), 84-92. (In chinese)

[4] Jiang Y. H., Pan Q. J., Tang J., Li Y. Integral control and low-pass control in adaptive interference cancellation system. Transactions of china electrotechnical society. 26, 12 (Dec. 2011), 148-155.

[5] Li W. L., Zhao Z. H., Tang J., He F. M., Li Y., Xiao H. Performance Analysis and Optimal Design of the Adaptive Interference Cancellation System. IEEE Transactions on Electromagnetic Compatibility. 55, 6 (Jun. 2013), 1068-1075.

[6] Yao Z. X., Li H. S., Ren G. X. The performance analysis of adaptive interference cancellation system. Journal of Xidian University. 22, 3 (Sep. 1995)256-261. (In chinese) 